\title{
Sex differences in performance and hemispheric organization for a nonverbal auditory task
}

\author{
GERALD W. MCROBERTS and BARBARA SANDERS \\ University of Connecticut, Storrs, Connecticut
}

\begin{abstract}
Musically experienced and inexperienced men and women discriminated among fundamentalfrequency contours presented either binaurally (i.e., same contour to both ears) or dichotically (i.e., different contours to each ear). On two separate occasions, males made significantly fewer errors than did females in the binaural condition, but not in the dichotic condition. Subjects with prior musical experience were superior to musically naive subjects in both conditions. The dichotic pitch task produced a left-ear advantage, which was unrelated to gender or musical experience. The results suggest that the male advantage on the binaural task reflects a sex difference in the coordination of the two hemispheres during conjoint processing of the same stimuli rather than a difference in the direction or degree of hemispheric specialization for these stimuli.
\end{abstract}

Lateralized stimulus presentation (e.g., dichotic listening or lateralized visual-field presentation) has become established as the basic means of measuring differences in hemispheric organization among normal subjects (e.g., Segalowitz \& Bryden, 1983). In the dichotic listening paradigm, auditory signals that differ on some theoretically relevant dimension (e.g., voice-onset time for consonant-vowel (CV) syllables or the frequencies of constituent members of a chord) are presented simultaneously to the two ears. The listener's task is to identify (label) the stimulus in a designated ear (or both ears). Significant differences in performance between the two ears are typically interpreted as reflecting an advantage for the contralateral hemisphere in processing information along the stimulus dimension on which the stimuli differ. Thus, a right-ear advantage for dichotically presented CV syllables is typically interpreted as reflecting the advantage of the left hemisphere for language and speech-related activities (e.g., Geffen \& Quinn, 1984; Kimura, 1961; Studdert-Kennedy \& Shankweiler, 1970). Differences between groups of normal subjects (e.g., males and females, right- and left-handers, or musicians and nonmusicians) on these laterality measures have been interpreted as indicating differences in neurological organization, or cognitive strategy. Negative findings are more difficult to interpret, but they have typically been regarded as evidence for no difference in neurological organization and/or cognitive strategies between groups. However, testing with lateralized stimulus presentation (whether in the auditory or visual modality) measures only the relative abilities of the two hemispheres to operate independently or in competition. This leaves untested the ability of the two hemispheres to operate conjointly to solve the same task (e.g., Allen, 1983).

Correspondence should be addressed to G. W. McRoberts, who is now at the Department of Psychology, Stanford University, Stanford, CA 94305-2130.
Since stimulus presentation outside the laboratory is seldom lateralized, the demonstration of differences in lateralization between two groups may be largely irrelevant to how perceptual and cognitive functioning operates in the world outside the laboratory. A more interesting approach might ask whether differences in lateralization reflect differences in cognitive processes under more normal conditions, such as when the two hemispheres operate conjointly. To assess the relations between the independent and conjoined functioning of the two hemispheres, it is necessary to test subjects with the same stimuli presented in both lateralized and nonlateralized conditions. Thus, both binaural testing and dichotic testing (or central presentation and lateralized-field presentation, in the visual modality) are necessary to provide a picture of the functioning of the two hemispheres in both independent and conjoined conditions.

In a study of fundamental-frequency contour identification (McRoberts, Sanders, \& Studdert-Kennedy, 1983), we utilized both dichotic and binaural stimulus presentation. Subjects labeled fundamental-frequency contours on the basis of the direction of change in the contour (i.e., rising, falling, rising-falling, falling-rising). During a binaural training task used to acquaint the subjects with the pitch contour task, males were significantly more accurate than females. However, on dichotic tests given on 2 separate days, there were no significant differences between males and females in either performance or ear advantage (both sexes showed a significant left-ear advantage).

Our result, which indicated a sex difference for nonlateralized stimulus presentation but not for lateralized presentation, is mirrored by a recent study of haptic pattern recognition by Cohen and Levy (1986). In this study, bimanual and dihaptic trials were interspersed. On dihaptic trials, subjects were presented with geometric designs made of abrasive paper to the target hand and random tactile interference to the nonreported hand. Under bimanual stimulation, both hands explored the geometric shapes. 
For the dihaptic trials, males and females were equally accurate and both demonstrated equal left-hand advantages. However, on the bimanual trials, males performed better than females.

Cohen and Levy's bimanual and dihaptic trials are analogues of our binaural and dichotic conditions, respectively. In each study, the same or different stimuli are presented to the two sensory hemifields (ears or hands), and in both studies, sex differences occurred in bifield stimulation, but not in difield stimulation.

The present study was carried out to document, under more systematic conditions, our previous finding of a sex $x$ stimulus-presentation condition interaction. We tested all subjects on 2 separate days, first in a binaural-dichotic test order and then in the reverse order. (Since the dichotic pitch contour task required some binaural experience to be performed, a completely counterbalanced design could not be used).

We included the Seashore Pitch Discrimination and Tonal Memory Tests in our study as comparison tasks for the pitch contour task, because sex differences have not, to the best of our knowledge, been reported on these binaurally presented tests. We also controlled for the effects of handedness and family handedness, while systematically varying one specific experience-musical trainingwhich has been related to both performance and lateralization of some auditory tasks (e.g., Bever \& Chiarello, 1974; Gordon, 1975, 1978a, 1978b).

\section{METHOD}

\section{Design}

Musically experienced and musically naive males and females were tested on a fundamental-frequency contour identification task in a repeated measures design that included binaural and dichotic stimulus-presentation conditions counterbalanced on each of 2 separate days (binaural/dichotic on Day 1, dichotic/binaural on Day 2). The Seashore Pitch Discrimination and Tonal Memory Tests were administered on the first day, before exposure to the pitch contours.

\section{Subjects}

The subjects were 24 males and 24 females from introductory psychology courses at the University of Connecticut. Half of the subjects of each sex were selected as musically experienced and half were selected as musically naive on the basis of their responses to questionnaires regarding musical training and handedness/familial handedness. Inclusion in the musically experienced group required formal musical training and experience with a musical instrument continuously since age 12 . The majority of subjects in this group were music majors. Most indicated proficiency with more than one instrument, and all indicated the ability to read music. The average number of years of formal musical training did not differ between the males and females in the musically trained group (6.75 vs. 7.25 years). The criterion for inclusion in the musically naive group was a report of total absence of formal musical training or experience in playing an instrument. All subjects were strongly righthanded, as indicated by responses on a nine-item hand-use questionnaire. No subject reported a left-handed (or ambidextrous) parent or sibling.

\section{Tests}

Seashore tests. In the Seashore Pitch Discrimination Test, 50 pairs of tones are presented. For each tone pair, the listener is to decide whether the second tone is higher or lower in pitch than the first. The stimuli in this test are pairs of pure tones derived from a beat-frequency oscillator. The tones have a frequency of about $500 \mathrm{~Hz}$ and a duration of $600 \mathrm{msec}$ each. Frequency differences between the tones in the pairs range from 17 to $2 \mathrm{~Hz}$ (Seashore, Lewis, \& Saetveit, 1960).

The Seashore Tonal Memory Test consists of 30 pairs of tonal sequences, including 10 pairs each of three-, four-, and five-tone sequences. In each pair, one note is different in the two sequences. The subject is to specify by number which tone is different (i.e., first, second, etc.). A Hammond organ was used as the stimulus source. The 18 chromatic steps upward from middle $C$ were used. Tempo was carefully controlled, and intensity was essentially constant (Seashore et al., 1960).

Fundamental-frequency contour tests. The stimuli for the binaural and dichotic fundamental-frequency contour tests were computer-generated at Haskins Laboratories. Each stimulus was a complex waveform composed of a fundamental frequency $(F 0)$ and two stable formants at approximately 3500 and $5400 \mathrm{~Hz}$, with a duration of approximately $250 \mathrm{msec}$. The $F 0$ contours were rising, falling, rising-falling, and falling-rising. To avoid problems of masking with dichotic presentation, each contour occurred in two frequency ranges. In dichotic presentation, a token from the low-frequency range was always paired with a different token from the high-frequency range. The contours in the low-frequency range were centered around a fundamental frequency of approximately $100 \mathrm{~Hz}$ and changed over approximately $30 \mathrm{~Hz}$. Thus, for example, the rising $F 0$ contour began at about $85 \mathrm{~Hz}$ and ended at about $115 \mathrm{~Hz}$, whereas the rising-falling contour also began at about $85 \mathrm{~Hz}$, rose to $115 \mathrm{~Hz}$, and returned to $85 \mathrm{~Hz}$. The contours in the high range were of the same nature but had a higher fundamental frequency. In binaural presentation, equal numbers of stimuli were included from each frequency range.

\section{Procedure}

The subjects were tested in groups of 3-5 in two separate 1-h sessions, approximately $48 \mathrm{~h}$ apart. Tests were administered to all subjects in the same order.

Day 1. The subjects were first tested on the Seashore Pitch Discrimination and Tonal Memory Tests, followed by the binaural and dichotic $F 0$ contour tests (in that order). Before the binaural test, the subjects were familiarized with the $F 0$ contours and the response to be associated with each contour. The contours were represented by oblique lines that matched the direction of the $F 0$ contour (e.g., $/, \backslash, \wedge, \vee)$. The subjects listened twice to a demonstration stimulus sequence presented binaurally over headphones while the experimenter indicated which response went with each $F O$ contour. Twenty-four binaural trials were then presented in which the experimenter indicated the correct response on each trial, followed by two 12-trial blocks in which the subjects responded on a prepared answer sheet by circling the appropriate contour. Feedback was given after each block. The binaural test followed the familiarization phase and was composed of $\mathbf{4 8}$ trials presented in four blocks of 12 trials each. The test included equal numbers of each stimulus token. After the binaural test, a five minute break occurred.

Before the dichotic test, two blocks of 12 dichotic practice trials were presented. The subjects were instructed to respond to the right ear for the first block and to the left ear for the second block. Feedback was not provided. On the dichotic test, order of ear report was $R-L-L-R$ or $L-R-R-L$ and was counterbalanced across the four sex $\times$ musical training groups. The dichotic test was composed of 96 dichotic trials presented in eight blocks of 12 trials each. Fortyeight trials, including equal numbers of each stimulus token, were reported from each ear. The subjects were instructed in advance to attend to either the right or the left ear for two consecutive blocks (24 trials). They were then instructed to attend to the opposite ear for two blocks ( 24 trials). Earphones were then reversed, and the subjects were instructed to continue attending to the same ear for 
Table 1

Percent Correct on the Seashore Tests

\begin{tabular}{|c|c|c|c|c|c|c|}
\hline \multirow[b]{2}{*}{ Seashore Test } & \multicolumn{3}{|c|}{ Males } & \multicolumn{3}{|c|}{ Females } \\
\hline & $\begin{array}{c}\text { Musically } \\
\text { Experienced }\end{array}$ & $\begin{array}{c}\text { Musically } \\
\text { Naive }\end{array}$ & Totals & $\begin{array}{c}\text { Musically } \\
\text { Experienced }\end{array}$ & $\begin{array}{l}\text { Musically } \\
\text { Naive }\end{array}$ & Means \\
\hline $\begin{array}{l}\text { Pitch Discrimination } \\
\text { Tonal Memory }\end{array}$ & $\begin{array}{l}85.5 \\
87.2\end{array}$ & $\begin{array}{l}76.7 \\
76.1\end{array}$ & $\begin{array}{l}81.1 \\
81.6\end{array}$ & $\begin{array}{l}85.5 \\
83.9\end{array}$ & $\begin{array}{l}80.2 \\
78.6\end{array}$ & $\begin{array}{l}82.8 \\
81.2\end{array}$ \\
\hline Means & 86.3 & 76.4 & 81.4 & 84.7 & 79.4 & 82.0 \\
\hline
\end{tabular}

an additional two blocks. The subjects were then instructed to attend to the opposite ear for the final two blocks.

Day 2. On the second day of testing, the subjects were refamiliarized with the $F 0$ contours by listening to 24 trials presented binaurally without feedback. The dichotic test was then administered, followed, after a 5-min interval, by the binaural test. Order of ear report on the dichotic test was consistent from Day 1 to Day 2.

\section{RESULTS}

\section{Musical Training, Pitch Discrimination, and Tonal Memory}

The performance of musically experienced and naive males and females on the two Seashore tests is shown in Table 1. A repeated measures analysis of variance (ANOVA), with sex $\times$ musical training, on the percent correct on each of the two Seashore tests indicated that, overall, musically experienced subjects performed significantly better than musically naive subjects $[F(1,44)=$ $6.107, p<.05]$. The performance of males and females did not differ $[F(1,44)=.047]$. No interactions were significant. The Seashore Pitch Discrimination and Tonal Memory Tests were correlated $r=.55(p<.0001)$.

\section{Fundamental-Frequency Contours}

Mean performance by males and females on the pitch contour test is shown in Figure 1. A repeated measures ANOVA (sex $\times$ musical training) was performed on the percent correct in each condition on each day (1 and 2). As predicted, the sex $\times$ condition interaction $[F(1,44)=$ $8.92, p<.01]$ was significant. Simple effects tests of

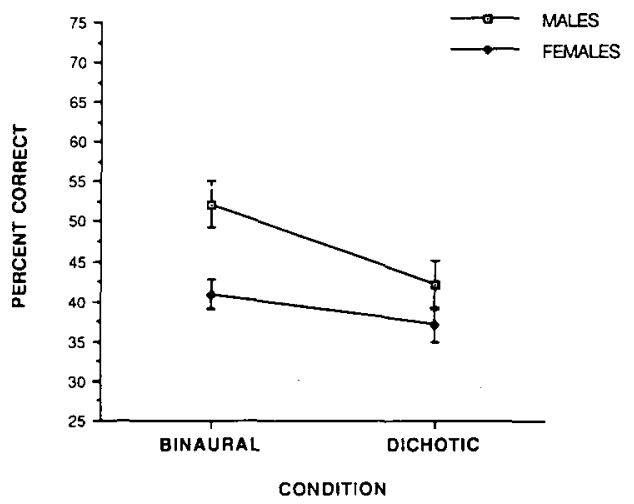

Figure 1. Performance in the binaural and dichotic experimental conditions for male and female listeners. this interaction indicate that the males were superior to the females in the binaural condition $[F(1,44)=12.88$, $p<.01]$, but not in the dichotic condition $[F(1,44)=$ $2.67, p>.10]$.

The repeated measures analysis also indicated that, overall, the males were more accurate than the females [sex, $F(1,44)=7.64, p<.01]$, musically experienced subjects were more accurate than musically naive subjects [musical training, $F(1,44)=6.86, p<.05$ ], more stimuli were correctly identified in the binaural condition than in the dichotic condition [condition, $F(1,44)=$ $45.34, p<.001$ ], and there was a practice effect [day, $F(1,44)=13.13, p<.01]$. However, a condition $\times$ day interaction $[F(1,44)=8.38, p<.01]$ also occurred, reflecting the fact that more improvement was made on the binaural test than on the dichotic test. The sex $\times$ condition $\times$ day interaction was not significant $[F(1,44)=$ $3.30, p>.05$ ]

Examination of the number correct for each ear revealed that there was a left-ear advantage on the dichotic test, the magnitude of which was unrelated to sex or musical training. Although the mean ear advantage was small, it was comparable in magnitude to the right-ear advantage often reported for CV syllables, which are typically not large and differ as a function of the specific stimuli used (e.g., Studdert-Kennedy \& Shankweiler, 1970). A repeated measures ANOVA (sex $\times$ musical training) performed on the scores from each ear on each dichotic test confirms these observations. Significant effects of ear $[F(1,44)=5.56, p<.05]$, musical training $[F(1,44)=$ $9.53, p<.01]$, and test $[F(1,44)=6.15, p<.05]$ indicate that, overall, there were more correct responses reported from the left ear than from the right ear, musically experienced subjects were more accurate than musically naive subjects, and there was a practice effect such that overall performance was better on the second day. The main effect of sex was nonsignificant $[F(1,44)=$ $3.84, p>.05$ ], as were the ear $\times$ sex and ear $\times$ musical training interactions. A sex $\times$ musical training repeated measures ANOVA performed on a laterality index, [(RE-LE)/(RE + LE) $\times 100$ ], computed independently for each subject on each dichotic test indicated no significant effects (Marshall, Caplan, \& Holmes, 1975).

Since there were no differences between males and females on the dichotic test, the male advantage on the binaural test does not appear to be due to differences in lateralization for the task. We therefore wished to relate left-ear performance on the dichotic test with performance 


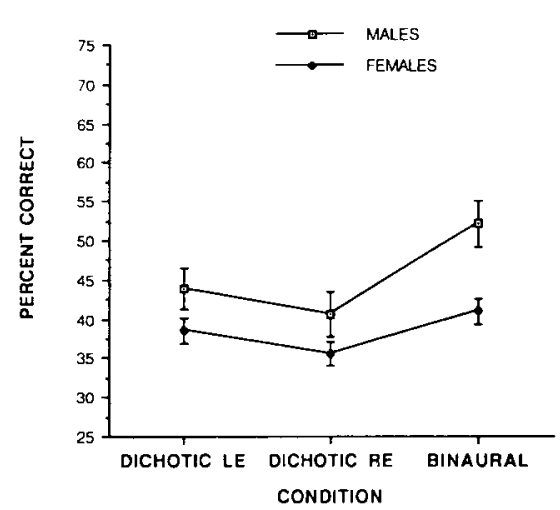

Figure 2. Comparison of lateralized and nonlateralized performance for male and female listeners.

on the binaural test. Mean percent correct for males and females on the dichotic and binaural tests is shown in Figure 2. Post hoc means tests were performed to compare left-ear performance with performance in the binaural condition for males and females separately. For males, performance in the binaural condition was significantly better than left-ear performance $[t(23)=5.95, p<$ $.0001]$; however, for females, the difference was not significant $[t(23)=1.541$, n.s. $]$.

\section{DISCUSSION}

The results of this study confirm our previous findings (McRoberts et al., 1983). Males correctly identified significantly more $F 0$ contours than did females when stimuli were presented binaurally; however, when the stimuli were presented dichotically, the sex difference was markedly reduced. The reduction in the sex difference is due to the fact that males' performance in the binaural condition was much superior to their dichotic performance, while females differed less between conditions.

The male advantage on the binaural task was substantial, accounting for nearly $15 \%$ of the total variance in performance in each test. Sex differences of this magnitude on perceptual-cognitive tasks are relatively rare (Hyde, 1981). Other research in Sanders' laboratory, has singled out one particular test of spatial ability, the Shepard/Metzler Mental Rotations Test, as one that produces an exceptionally large male advantage in comparison with other cognitive measures, including other measures of spatial ability (Sanders, Soares, \& D'Aquila, 1982). The male advantage on the Shepard/Metzler test is approximately the same magnitude as the male advantage in the binaural condition of the present study. As with spatial ability, the sex difference in the present study is fairly specific. No sex difference was found on the Seashore tests, both of which involve the binaural perception of differences between two or more tones of constant frequency. In the $F 0$ contour task, where the stimuli are frequency ramps or glides, listeners are required to detect the direction of movement within each stimulus.
Various studies have suggested that the perception of differences between constant frequency tones is not based on the same abilities as the perception of frequency glides (e.g., J. D. Harris, 1957; Kay \& Matthews, 1972; Sergeant \& J. D. Harris, 1962; Whitfield \& Evans, 1965).

A critical feature for interpreting the sex difference in the present study is that it appeared only when stimulus presentation was binaural rather than dichotic. This finding is strikingly similar to a result obtained by Cohen and Levy (1986) in a completely different stimulus modality. Using a haptic task, these investigators found a male advantage on bimanual trials, but not on dihaptic trials with the same stimuli. In their study, the sex difference during conjoint processing resulted from a reduction in female performance, whereas in our study, it was due to improved male performance. Nonetheless, both results indicate that a sex difference favoring males emerges when the hemispheres are required to work together in making a perceptual discrimination, rather than operating in relative isolation. The biological basis for this sex difference may be a difference in the relative width of the corpus collosum (Baack, de Lacoste-Utamsing, \& Woodward, 1982; de Lacoste-Utamsing \& Holloway, 1982; Nasrallah et al., 1985; Witelson, 1985).

A frequent explanation for sex differences in performance on perceptual-cognitive tasks is that males and females differ in the degree of hemispheric specialization for the task or some component task (e.g., L. Harris, 1978; Levy, 1976; Witelson, 1976). Thus, for example, the male superiority on spatial tasks is assumed to result from greater right-hemisphere specialization for mental rotations among males. Such an explanation predicts a difference in degree of asymmetry for tasks on which sex differences are found. However, in neither the current study nor the study by Cohen and Levy (1986) did males and females differ in the degree of ear advantage (or laterality index) for the $F 0$ contours. Therefore, it would appear that differences in right-hemisphere specialization cannot underlie these sex differences in performance. Rather, the results in these two studies suggest that males and females differ in performance when the two hemispheres are allowed to work in cooperation (i.e., both hemispheres receive the same information).

Suggestions that sex differences in perceptual-cognitive functions may have a biological basis are frequently countered by criticisms that the effects of experience are uncontrolled. Musical training is an important kind of auditory experience that might differ between the sexes and thus influence group differences. The present study represents a major methodological advance in the control of experience in studies of sex differences, since we equated years of musical training between the sexes. We find that the sex difference is independent of the influence of musical training in our sample. The magnitude of the sex difference is as large in the musically experienced group (1 standard deviation) as in the musically naive group (1 standard deviation). (Indeed, although musically experienced females perform better than musically naive fe- 


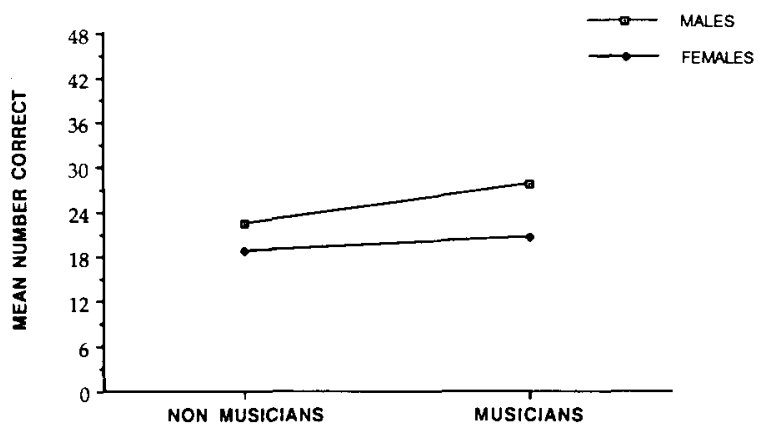

Figure 3. Performance in the binaural condition for male and female musicians and nonmusicians.

males, they only approach the performance of musically naive males. See Figure 3 ).

In addition, our results suggest that the effects of musical training are different from the effects of sex. Whereas musically experienced subjects performed better than did musically naive subjects on both Seashore tests and in both stimulus-presentation conditions with the $F 0$ contours, males and females differed only on the binaural $F 0$ contours test. Thus, the influence of musical training appears to be a more general effect influencing various auditory functions, whereas the influence of sex is specific to the F0 contours.

\section{REFERENCES}

ALlen, M. (1983). Models of hemispheric specialization. Psychological Bulletin, 93, 73-104.

BaAck, J., de Lacoste-Utamsing, C., Woodward, D. (1982). Sexual dimorphism in human fetal corpora callosa. Society for Neuroscience Abstracts, 8, 213.

Bever, T., Chiarello,C. (1974). Cerebral dominance in musicians and nonmusicians. Science, 185, 537-539.

Bouchard, T., \& McGee, M. (1977). Sex differences in human spatial ability: Not an X-linked recessive gene effect. Social Biology, 24, 332-335.

Cohen, H., Levy, J. (1986). Cerebral and sex differences in the categorization of haptic information. Cortex, 22, 253-260.

de Lacoste-Utamsing, C., \& Holloway, R. (1982). Sexual dimorphism in the human corpus collosum. Science, 216, 1431-1432.

GeFFEN, G., \& QUINN, K. (1984). Hemispheric specialization and ear advantages in processing speech. Psychological Bulletin, 96, 273-291.

GoRDON, H. (1975). Hemispheric asymmetry and musical performance. Science, 189, 60-69.

GoRDON, H. (1978a). Left hemispheric asymmetry for dichotically presented chords in musicians and nonmusicians, males and females. Acta Psychologia, 42, 383-395.

GoRdON, H. (1978b). Left hemisphere dominance for thythmic elements in dichotically-presented melodies. Cortex, 4, 58-70.

HARRIS, J. D. (1957). A search toward the primary auditory abilities. In $A$ decade of basic and applied science in the Navy. Washington, DC: U.S. Government Printing Office.

Harris, L. (1978). Sex differences in spatial ability: Possible environmental, genetic and neurological factors. In M. Kinsbourne (Ed.), Asymmetrical functions of the brain (pp. 405-522). New York: Cambridge University Press.

HYDE, J. (1981). How large are cognitive gender differences? American Psychologist, 36, 892-902.

KAY, R. H., MATthews, D. R. (1972). On the existence in human auditory pathways of channels selectively tuned to the modulation present in frequency-modulated tones. Journal of Physiology, 225, 657-677.

KimurA, D. (1961). Cerebral dominance in the perception of verbal stimuli. Canadian Journal of Psychology, 15, 166-171.

LEVY, J. (1976). Cerebral lateralization and spatial ability. Behavior Genetics, 6, 171-188.

Marshall, J., Caplan, D., \& Holmes, J. (1975). The measure of laterality. Neuropsychologia, 13, 315-321.

McRoberts, G. W., SANDERs, B., \& Studdert-KenNedy, M. (1983). Disconfirmation of Kinsboume's attentional hypothesis. Paper presented at the Eastern Psychological Association, Philadelphia, PA.

Nasrallah, H., andreasen, N., Olson, S., Coffman, J., Coffman, C., DUNN, V., EhrhardT, J. (1985). Absence of sexual dimorphism of the corpus collosum in schizophrenia: A magnetic resonance imaging study. Society for Neuroscience Abstracts, 11, 1308.

SANDERs, B., SoAres, M., \& D'Aquila, J. (1982). The sex difference on one test of spatial visualization: A nontrivial difference. Child $D e-$ velopment, 53, 1106-1110.

SERGEANT, R. L., HaRRIS, J. D. (1962). Sensitivity to unidirectional frequency modulation. Journal of the Acoustical Society of America, 34, 1625-1628.

SEASHORE, C., LEWIS, D., \& SAETVEIT, J. (1960). Seashore measures of musical talent (Revised). New York: Psychology Corporation.

Segalowttz, S. J., B Bryden, M. P. (1983). Individual differences in hemispheric representation of language. In S. Segalowitz (Ed.), Language functions and brain organization (pp. 341-372). New York: Academic Press.

Studdert-KenNedy, M., ShankWEILER, D. (1970). Hemispheric specialization for speech perception. Journal of the Acoustical Society of America, 48, 570-594.

Whitfield, I. C., Evans, E. F. (1965). Responses of auditory cortical neurons to stimuli of changing frequency. Journal of Neurophysiology, 28, 655-672.

WITELSON, S. (1976). Sex and the single hemisphere: Specialization of the right hemisphere for spatial processing. Science, 193, 425-427.

Witelson, $S$. (1985). The brain connection: The corpus collosum is larger in left handers. Science, 229, 665-668.

(Manuscript received September 17, 1990; revision accepted for publication September 27, 1991.) 